\title{
Electrons in a Periodic Magnetic Field Induced by a Regular Array of Micromagnets
}

\author{
P. D. Ye, ${ }^{1}$ D. Weiss, ${ }^{1}$ R. R. Gerhardts, ${ }^{1}$ M. Seeger, ${ }^{2}$ K. von Klitzing, ${ }^{1}$ K. Eberl, ${ }^{1}$ and H. Nickel ${ }^{3}$ \\ ${ }^{1}$ Max-Planck-Institut für Festkörperforschung, D-70569 Stuttgart, Germany \\ ${ }^{2}$ Max-Planck-Institut für Metallforschung, Institut für Physik, D-70569 Stuttgart, Germany \\ ${ }^{3}$ Forschungsinstitut der Deutschen Bundespost, D-64295 Darmstadt, Germany
}

(Received 2 December 1994)

\begin{abstract}
The deposition of ferromagnetic microstructures on top of a high-mobility two-dimensional electron gas (2DEG) allows the investigation of electron transport in a periodic magnetic field which alternates on a length scale small compared to the elastic mean free path of the electrons. The longitudinal resistance of the 2DEG displays, as a function of the externally applied field, the long-predicted magnetic commensurability oscillations which result from the interplay between the two characteristic length scales of the system, the classical cyclotron radius $R_{c}$ of the electrons and the period $a$ of the magnetic field modulation.
\end{abstract}

PACS numbers: 73.50.Jt, 73.20.Dx, 75.50.Rr

Transport properties of electrons in a two-dimensional electron gas (2DEG) subjected to a periodic magnetic field have attracted considerable theoretical interest [16]. Depending on the strength of the local magnetic field, the electron motion in the plane of the $2 \mathrm{DEG}$ can be tuned from regular to chaotic. The motion of ballistic electrons in a periodic magnetic field is also believed to be closely related to the motion of composite fermions in a density modulated 2DEG in the fractional quantum Hall regime [7]. Distinct theoretical predictions exist for the limit of a weak one-dimensional (1D) magnetic modulation (modulation amplitude $\left|B_{m}\right| \ll\left|B_{0}\right|$, the external magnetic field) where the magnetoresistance $\rho_{x x}$ is expected to oscillate with minima appearing at magnetic fields given by $[2-5]$

$$
2 R_{c}=\frac{2 \hbar k_{F}}{e\left|B_{0}\right|}=\left(\lambda+\frac{1}{4}\right) a,
$$

where $\lambda=0,1, \ldots$ is an integer oscillation index, $k_{F}=$ $\sqrt{2 \pi n_{s}}$ the Fermi wave number, with $n_{s}$ the carrier density of the 2DEG, and $a$ the period of the 1D modulation in the $x$ direction. This predicted effect is closely related to the commensurability oscillations observed recently in the resistivity $\rho_{x x}$ of a 2 DEG with weak electric modulation [8-10]. Similar to the electric case, the magnetic modulation leads to a modified energy spectrum [1-5]. The degenerate Landau levels are transformed into bands of finite width. The dispersion of these Landau bands provides an additional contribution to the resistivity $\rho_{x x}$, which vanishes only when the bandwidth becomes zero ("flat-band condition") $[9,10]$. In contrast to Eq. (1), which is the flat-band condition for magnetic modulation, the flat-band condition for weak electrical modulation reads $2 R_{c}=(\lambda-1 / 4) a$ with $\lambda=1,2, \ldots$. Hence, $\rho_{x x}$ of a 2 DEG with a weak electric modulation displays minima at $B_{0}$ fields where in a weak magnetic modulation of the same period $a$ maxima are expected.

For the case of a pure electric modulation, the additional contribution to $\rho_{x x}$ has been related to the classical guiding center drift of the cyclotron orbits which vanishes if the flat-band condition holds [11]. This classical picture is easily extended to include in addition to a modulation $V_{m}(x)=V_{m} \cos K x$ of the electrostatic potential energy of an electron a weak modulation $B_{m}(x)=B_{m} \cos K x$ of the $z$ component of the magnetic field $(K=2 \pi / a)$. Averaging the modulation induced drift of the guiding centers over the unperturbed cyclotron orbits at field $B_{0}[11,12]$, we obtain for the resulting change in the resistivity

$$
\frac{\Delta \rho_{x x}}{\rho_{0}}=\frac{2 \tau^{2}}{\pi \hbar^{2} n_{s} a R_{c}}|S|^{2},
$$

where $\rho_{0}$ is the zero-field resistivity of the unmodulated 2DEG, $\tau$ is the scattering time, and

$$
S=V_{m} \cos \left[K R_{c}-\frac{\pi}{4}\right] \pm \frac{k_{F} a}{2 \pi} \hbar \omega_{m} \sin \left[K R_{c}-\frac{\pi}{4}\right]
$$

with $\omega_{m}=e B_{m} / m^{*}$ ( $m^{*}$ is the effective electron mass of GaAs). The "+" in Eq. (3) holds if $B_{0}>0$, and the "-" sign holds if the applied field (and thus the direction of the cyclotron motion) is reversed, $B_{0}<0$, while $V_{m}(x)$ and $B_{m}(x)$ are fixed [13]. The zeros of Eq. (3) and hence the flat-band positions now depend on the relative strengths of electric and magnetic modulations, $V_{m}$ and $\hbar \omega_{m}$, respectively.

Over the last years a variety of experimental attempts were made (e.g., [5]) to establish a periodic magnetic field on the length scale of a few hundred nanometer, so far, however, without success. In this Letter we report a novel method to investigate electron transport in a periodic magnetic field. By depositing an array of ferromagnetic dysprosium (Dy) strips with widths of a few hundred nanometer on top of a semiconductor heterojunction (Fig. 1), we generate a 1D periodic magnetic field in the plane of the 2DEG. By increasing the strength of these micromagnets via the externally applied field we show that the long-desired magnetic commensurability oscillations appear in the resistivity $\rho_{x x}$. 


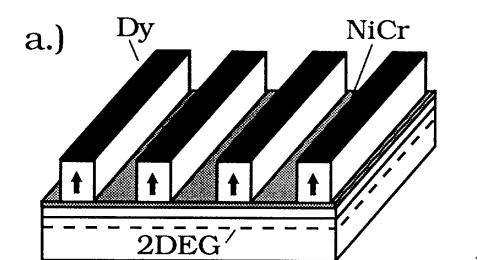

b.)

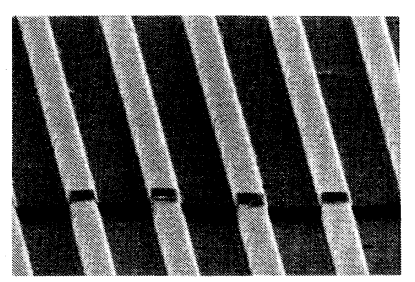

c.)

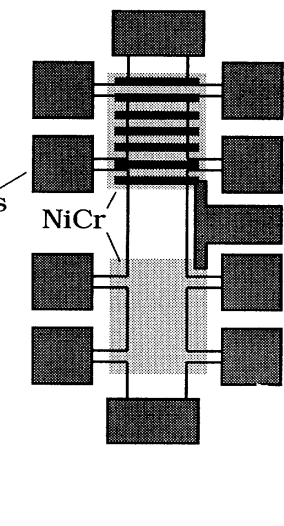

FIG. 1. (a) Sketch of the one-dimensional ferromagnetic Dy grating on top of a GaAs-AlGaAs heterojunction. (b) Electron micrograph of the Dy strips evaporated across a mesa edge: $a=1 \mu \mathrm{m}$, height of a Dy strip: $200 \mathrm{~nm}$. (c) Device geometry containing the ferromagnetic grating and an unpatterned reference Hall bar.

Our samples were prepared from high-mobility GaAsAlGaAs heterojunctions where the 2DEG was located approximately $100 \mathrm{~nm}$ underneath the sample surface. The carrier density $n_{s}$ and electron mobility $\mu$ at $4.2 \mathrm{~K}$ were $\sim 2.2 \times 10^{11} \mathrm{~cm}^{-2}$ and $1.3 \times 10^{6} \mathrm{~cm}^{2} / \mathrm{V} \mathrm{s}$, respectively, corresponding to an elastic mean free path of $\sim 10 \mu \mathrm{m}$. $50 \mu \mathrm{m}$ wide Hall bars, sketched in Fig. 1(c), were fabricated by standard photolithographic techniques. $\mathrm{Al}$ loyed $\mathrm{AuGe} / \mathrm{Ni}$ pads contact the 2DEG. A $10 \mathrm{~nm}$ thin $\mathrm{NiCr}$ film, evaporated on top of the devices, defines an equipotential plane to avoid electric modulation of the 2DEG. However, strain due to different thermal expansion coefficients of the ferromagnetic grating and the heterojunction always results in a weak electric periodic potential as the sample is cooled down to cryogenic temperatures (see below). The Dy gratings with period of $500 \mathrm{~nm}$ and $1 \mu \mathrm{m}$ were defined by electron beam lithography on top of one of the $\mathrm{NiCr}$ gates. After developing the exposed PMMA resist a $200 \mathrm{~nm}$ Dy film was evaporated. After lift-off in acetone micromagnet arrays, like the one shown in Fig. 1(b), were obtained. For magnetization measurements, large area $\left(5 \times 5 \mathrm{~mm}^{2}\right)$ Dy wire arrays with comparable wire width were prepared by holographic lithography. Four point resistance measurements were performed in a ${ }^{4} \mathrm{He}$ cryostat with superconducting coils using standard ac lock-in techniques. For all experiments, the external magnetic field $B_{0}$ was applied normal ( $z$ direction) to the plane of the 2DEG.

Measurements of the macroscopic stray fields of the large array of holographically prepared Dy wires $(a=$ $760 \mathrm{~nm}$ ) were measured with a Quantum Design SQUID magnetometer. The resulting hysteresis loop shown in Fig. 2 indicates hard magnetic behavior of the Dy wires. The coercive field $B_{c}$ of $0.5 \mathrm{~T}$ is high compared to pure polycrystalline Dy $\left(B_{c}<50 \mathrm{mT}\right)$. Hence, the demagne-

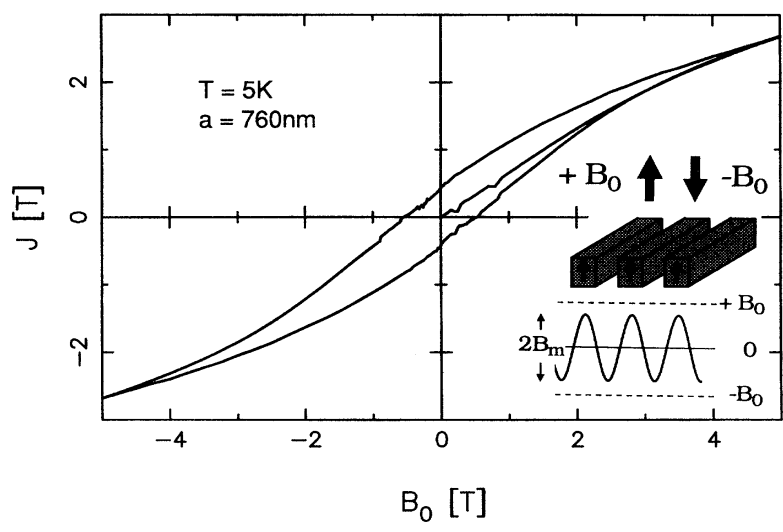

FIG. 2. Hysteresis of the magnetic polarization $J$ measured from a large array $\left(5 \times 5 \mathrm{~mm}^{2}\right)$ of Dy wires prepared by holographic lithography on a GaAs substrate. The inset sketches the normal component $B_{m}(x)$ of the stray field induced by the micromagnets in the plane of the $2 D E G$. Reversing the external magnetic field results in a phase shift of the modulation with respect to the grating (see text).

tization process is controlled either by the pinning or the nucleation mechanism [14]. The stray field $B_{m}(x)$ in the $z$ direction, sketched in the inset of Fig. 2, is expected to alternate with an average value close to zero [4]. We return to this point below.

Figure 3(a) shows the resistivity $\rho_{x x}$ of the 2DEG underneath the Dy superlattice with period $a=1 \mu \mathrm{m}$. Since the devices with $a=500 \mathrm{~nm}$ display comparable behavior, we concentrate on the larger period sample below. The traces labeled $1-10 \mathrm{~T}$ are obtained as follows: After the initial cooldown we first sweep to $1 \mathrm{~T}$ and measure the $\rho_{x x}$ trace labeled $1 \mathrm{~T}$ from 1 to $-0.25 \mathrm{~T}$. Then we sweep to $B^{\max }=2 \mathrm{~T}$ and take the next $\rho_{x x}$ trace in the same $B_{0}$ interval. By successively sweeping to higher $B^{\max }$ (up to $10 \mathrm{~T}$ ) the magnetic polarization $J$ in the Dy strips and hence the strength of the resulting periodic field $B_{m}(x)$ are increased. This enhanced strength affects the magnetoresistance traces: $\rho_{x x}$ displays oscillations with a dramatically growing maximum between $B_{0}=0.15$ and $0.75 \mathrm{~T}$ (the superimposed oscillations above $0.5 \mathrm{~T}$ are Shubnikov-de Haas oscillations). For positive $B_{0}$, the minima in $\rho_{x x}$ appear at $B_{0}$ values expected from Eq. (1) for magnetic modulation [15]. From the amplitude $\Delta \rho_{x x}$ of the maxima at $B_{0} \sim 0.3 \mathrm{~T}$ we estimate the amplitude of the imposed magnetic field $B_{m}$ from Eq. (2) with $2 R_{c} \approx 0.75 a$. This gives $B_{m}$ values, plotted in the inset of Fig. 3(a), which range between $13 \mathrm{mT}$ for the $B^{\max }=1 \mathrm{~T}$ trace and $40 \mathrm{mT}$ for the $B^{\max }=10 \mathrm{~T}$ trace. At low $B_{0}$ the traces are not symmetric with respect to $B_{0}=0$ as is expected for a broken time reversal symmetry [16]. This can be seen clearly in Fig. 3(b) where the low $B_{0}$ regime of Fig. 3(a) is magnified. With increasing $B^{\max }$ a richer oscillatory structure unfolds in $\rho_{x x}$ indicating a growing magnetic field modulation. The $\rho_{x x}$ minima for $B_{0}>0$ of trace $e$ almost perfectly coincide 

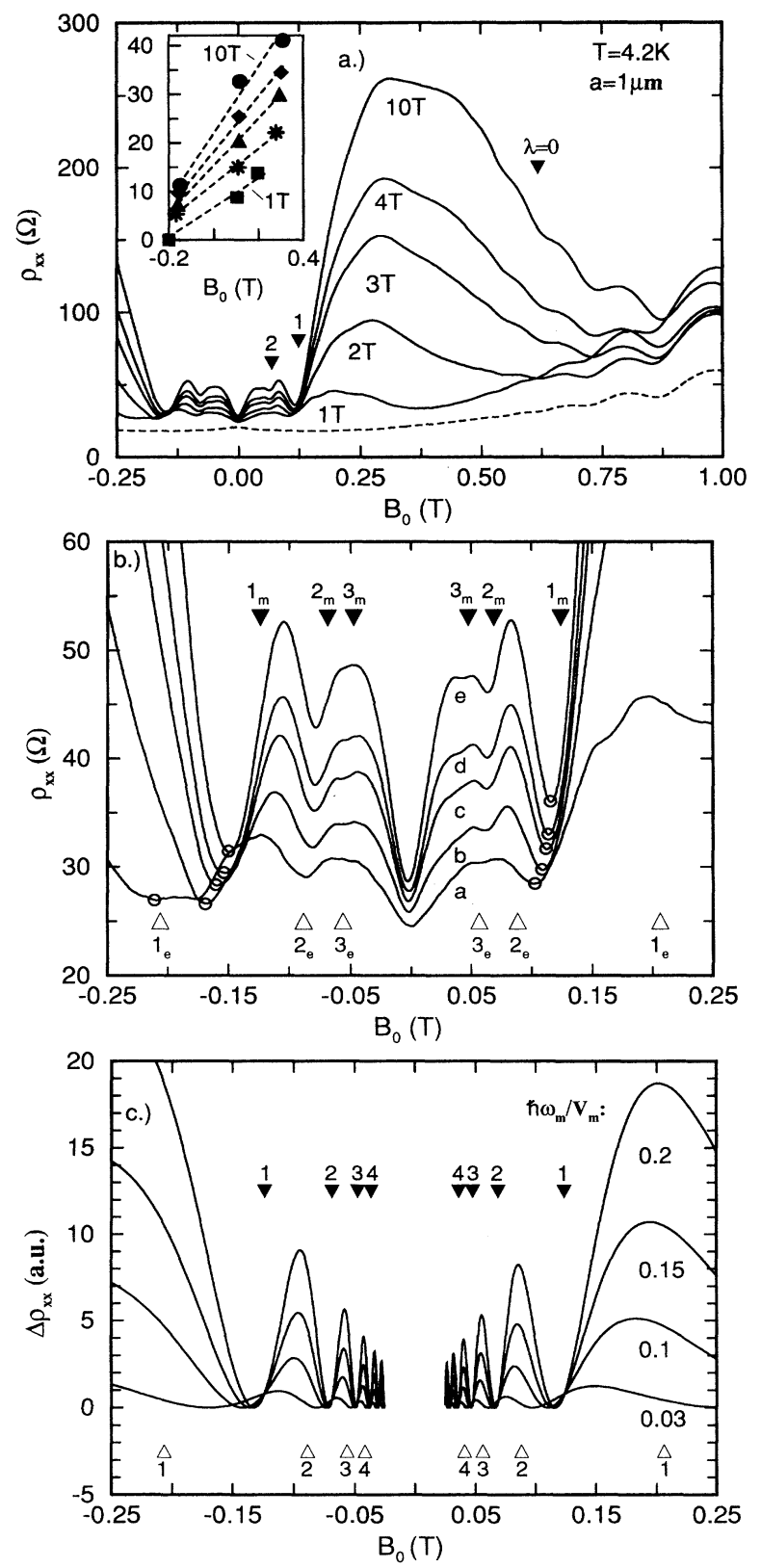

FIG. 3. (a) $\rho_{x x}$ vs external magnetic field $B_{0}$ for different $B^{\max }$ sweeps. Filled triangles with positions defined by Eq. (1) mark the flat-band condition in a periodic magnetic field. The inset displays the strength of the magnetic modulation $B_{m}$ (in $\mathrm{mT}$ ) as a function of $B_{0}$, derived from (i) the amplitude of the large resistance maximum at $\sim 0.3 \mathrm{~T}$ and (ii) the positions of the $\rho_{x x}$ minima in (b) around -0.16 and $0.12 \mathrm{~T}$. (b) Low-field magnification of (a) showing the shift of the $\rho_{x x}$ minima with increasing $B^{\max }$ (from $a=1 \mathrm{~T}$ to $e=10 \mathrm{~T}$ ). Filled triangles mark the position of the magnetic flat-band condition (subscript " $m$ ") while the open triangles mark the electric ones (subscript " $e$ "). The open circles highlight the positions of the $\rho_{x x}$ minima used to evaluate the $B_{m}$ 's from the zeros of Eq. (3). (c) $\Delta \rho_{x x}$ calculated for different $\hbar \omega_{m} / V_{m}$ ratios using Eq. (2). Filled and open triangles again mark magnetic and electric flat-band conditions. with what is expected from Eq. (1), indicating that the periodic magnetic field dominates over the weak electric potential. These are the long predicted magnetic commensurability oscillations.

The polarization $J$ (Fig. 2) and hence the stray field $B_{m}(x)$ of the micromagnets depend (besides on $B^{\max }$ ) on $B_{0}$. Therefore one expects to reduce $B_{m}$ by sweeping toward the coercive field $B_{c}$ around $-0.5 \mathrm{~T}$ [17]. However, following trace $a$ in Fig. 3(b) to negative $B_{0}$ one still observes oscillations in $\rho_{x x}$. But now the minima in $\rho_{x x}$ $(\lambda=1,2)$ appear at $B_{0}$ positions where one expects minima for pure electric modulation with period $a$, marked by open triangles. The origin of this electric modulation is strain caused by the different thermal expansion coefficients of Dy and GaAs. This effect, reported previously for other material combinations [5,18,19], results in a modulation of the band edges in GaAs via the deformation potential. Assuming a sinusoidal modulation we estimate from the amplitude of the resistance maximum at $B_{0} \sim-0.12 \mathrm{~T}$ (trace $\left.a\right)$ an amplitude of the (screened) electric potential $V_{m}$ of $\sim 0.3 \mathrm{meV}$ [20]. The presence of the weak electric modulation which is independent of $B^{\max }$ allows one to "calibrate" the strength of the stray field $B_{m}$. If $B^{\max }$ and hence $B_{m}$ are increased successively (traces $b-e$ ) all the $\rho_{x x}$ minima shift from the electric flatband condition (open triangles) to the magnetic one (full triangles). If both a periodic potential and an oscillating magnetic field act on the electrons, the flat-band case is determined by the zeros of Eq. (3). Since the position of the flat-band condition depends on the ratio $\hbar \omega_{m} / V_{m}$, $\hbar \omega_{m}$ and hence the field $B_{m}$ of the micromagnets can be estimated from the $B_{0}$ position of the $\rho_{x x}$ minima. The result is shown in the inset of Fig. 3(a). To obtain the $B_{m}$ 's we analyzed the position of the minima between $1_{e}$ and $1_{m}$ (here, the subscripts of the oscillation index $\lambda, e$ and $m$, stand for the electric and the magnetic flat-band conditions, respectively) for reversed $B_{0}$, and between $2_{e}$ and $1_{m}$ for positive $B_{0}$. The inset also includes the $B_{m}$ values estimated independently from the $\rho_{x x}$ maxima between $1_{m}$ and $0_{m}$. These $B_{m}$ values increase linearly with $B_{0}$, as is expected from the hysteresis trace in Fig. 2 in this magnetic field range.

The peculiar asymmetry for forward and reverse magnetic fields in Fig. 3(b) is also observed for the minima with higher $\lambda$ 's. The origin of this asymmetry is the switching of the " \pm " in Eq. (3) if $B_{0}$ is reversed. This change of the sign at $B_{0}=0$ can be viewed as a phase jump of $\pi$ between the electric modulation $V_{m}(x)$ and the total magnetic field strength $B(x)=\left|B_{0}+B_{m}(x)\right|$. This is illustrated in the inset of Fig. 2. Here, we put the $z$ axis in the direction of $B^{\max }$ and take $x=0$ in the center of a Dy strip, so that $B_{m}>0$. While the phase of the electric modulation (with respect to the ferromagnetic grating) is independent of the $B_{0}$ direction, the phase of the periodic $B$ field is not: for $B_{0}>0$ the local maxima of the $B$ field are underneath the Dy strips while for $-B_{c}<B_{0}<0$ the 
maxima are between the strips. This allows an absolute measurement of the phase of the electric modulation: Since the sign in Eq. (3) is "+" for $B_{0}>0$ while $B_{m}>0$, we obtain the correct shift of the minima for $V_{m}<0$, i.e., if $n_{s}$ is higher underneath the strips [21].

In Fig. 3(c) we display model calculations of $\rho_{x x}$ based on Eq. (2). The $\Delta \rho_{x x}$ oscillations calculated for four different $\hbar \omega_{m} / V_{m}$ ratios closely resemble the experimental data in Fig. 3(b). As in the experiment the oscillation minima shift with increasing $B_{m}$ from the electric flatband condition to the magnetic flat-band condition. For $\hbar \omega_{m} / V_{m}=0.2$, corresponding to $B_{m} \sim 40 \mathrm{mT}$ in our experiment, the $\rho_{x x}$ minima are determined by the magnetic flat-band conditions (filled triangles) indicating the dominance of the periodic $B_{m}$ field. The asymmetry with respect to the shift of the minima (see, e.g., the shift from $1_{e} \rightarrow 1_{m}$ for $B_{0}<0$ and from $2_{e} \rightarrow 1_{m}$ for $B_{0}>0$ with increasing $B_{m}$ ) is adjusted to the experiment by choosing $V_{m}<0$ (see above). For the sake of simplicity we used, in contrast to experiment, a $B_{0}$-independent magnetic field modulation $B_{m}$ to demonstrate the shift of the $\rho_{x x}$ minima.

If the reversed field is increased beyond the coercive field, the polarization of the micromagnets switches and finally follows the externally applied field. On sweeping back where we start from the corresponding $-B^{\max }$ values we obtain, as is expected from the hysteresis loop (Fig. 2), the mirror image (with respect to the $B_{0}=0$ axis) of the experimental traces shown above.

Now we address the zero-field resistance $\rho_{x x}\left(B_{0}=\right.$ $0)$ and the positive magnetoresistance at $\left|B_{0}\right|<$ $30 \mathrm{mT}$. First we note that the minimum of $\rho_{x x}$ at $B_{0}=0$ in Fig. 3(b) does not shift with increasing $B^{\max }$ (shift less than $3 \mathrm{mT}$ ) while, on the other hand, the amplitude $B_{m}$ becomes as high as $20 \mathrm{mT}$ at $B_{0}=0$ [see inset of Fig. 3(a)]. Such behavior is expected for an alternating stray field $B_{m}(x)$ with an average value of zero (inset of Fig. 2). This is consistent with the experimental finding that, independent of $B^{\max }$, the Hall resistance measured in the magnetic superlattice is the same as in the reference Hall bar. The positive low-field magnetoresistance might also be related to this alternating $B_{m}(x)$ field: electron trajectories "wiggling" along a $B_{m}(x)=0$ borderline (open orbits; for the electric case see, e.g., [22]) cause an increasing magnetoresistance which saturates once $\left|B_{0}\right|>\left|B_{m}\right|$. For trace $e$ in Fig. 3(b) the positive magnetoresistance saturates around $28 \mathrm{mT}$ comparable to the $B_{m}$ value of about $20 \mathrm{mT}$ in the inset of Fig. 3(a). Finally, we note that $\rho_{x x}(0)$ increases linearly with $B_{m}(0)$, as can be extracted from Fig. 3(b) and the inset of Fig. 3(a). Similar behavior is expected for electrons scattering from microinhomogeneities in a magnetic field [23].

In summary, we have observed the long-predicted commensurability oscillations in the presence of a periodic magnetic field. The experimental technique described above opens up the way to experiments in alternating magnetic fields with periods in the nanometer regime.
During the preparation of this manuscript we became aware of similar experiments using patterned superconductors on top of a heterojunction [24].

We thank M. Riek and A. Gollhardt for technical support, and W. Dietsche, H. Kronmüller, J. Smet, and M. Tornow for valuable discussions. P. D. Ye acknowledges the Volkswagen Foundation for a fellowship.

[1] D. Yoshioko and Y. Iye, J. Phys. Soc. Jpn. 56, 448 (1987).

[2] P. Vasilopoulos and F. M. Peeters, Superlattices Microstruct. 7, 393 (1990).

[3] F. M. Peeters and P. Vasilopoulos, Phys. Rev. B 47, 1466 (1993).

[4] D. P. Xue and G. Xiao, Phys. Rev. B 45, 5986 (1992).

[5] R. Yagi and Y. Iye, J. Phys. Soc. Jpn. 62, 1279 (1993).

[6] X. Wu and S.E. Ulloa, Solid State Commun. 82, 945 (1992); G. J. O. Schmidt, Phys. Rev. B 47, 13007 (1993); R. B.S. Oakeshott and A. MacKinnon, J. Phys. Condens. Matter 5, 9355 (1993); P. Schmelcher and D. L. Shepelyansky, Phys. Rev. B 49, 7418 (1994).

[7] W. Kang, H. L. Störmer, L. N. Pfeiffer, K. W. Baldwin, and K. W. West, Phys. Rev. Lett. 71, 3850 (1993); R. L. Willet, R. R. Ruel, K. W. West, and L. N. Pfeiffer, Phys. Rev. Lett. 71, 3846 (1993).

[8] D. Weiss, K. von Klitzing, K. Ploog, and G. Weimann, Europhys. Lett. 8, 179 (1989).

[9] R. R. Gerhardts, D. Weiss, and K. von Klitzing, Phys. Rev. Lett. 62, 1173 (1989).

[10] R. W. Winkler, J. P. Kotthaus, and K. Ploog, Phys. Rev. Lett. 62, 1177 (1989).

[11] C. W. J. Beenakker, Phys. Rev. Lett. 62, 2020 (1989).

[12] R. R. Gerhardts, Phys. Rev. B 45, 3449 (1992).

[13] For $B_{0}>0$ and intermediate temperatures $\left(T_{c} \ll T \ll T_{a}\right.$ in the notation of Ref. [3]) Eq. (23) of Ref. [3] is equivalent with our Eq. (2). Because of an incorrect formula for the diffusion tensor, the value given by Eq. (16) of Ref. [4] (for $V_{m}=0$ ) is too small by a factor of 2 .

[14] H. Kronmüller, K.-D. Durst, and M. Sagawa, J. Magn. Magn. Mater. 74, 291 (1988).

[15] The flat-band positions were derived using first order perturbation theory involving the cosine approximation of the Bessel functions $[11,12]$. Deviations are therefore expected for large values of $B_{0}$. This especially affects the $\lambda=0$ position in Fig. 3(a).

[16] To account for inhomogeneities in our devices we averaged over $\rho_{x x}$ traces taken from potential probes of both sides of the Hall bar.

[17] This is strictly valid only for $B^{\max }=5 \mathrm{~T}$. We expect $B_{c}$ values around $-0.5 \mathrm{~T}$ for the other traces.

[18] J.H. Davies and I. A. Larkin, Phys. Rev. B 49, 4800 (1994).

[19] P. D. Ye et al. (unpublished).

[20] D. Weiss, Phys. Scr. T35, 226 (1991).

[21] GaAs is expected to shrink with respect to Dy if cooled down, resulting in minima of $V_{m}(x)$ under the strips.

[22] P. H. Beton et al., Phys. Rev. B 42, 9229 (1990).

[23] A. V. Khaetskii, J. Phys. Condens. Matter 3, 5115 (1991).

[24] A. K. Geim (private communication). 


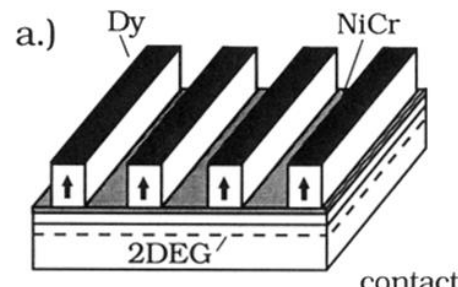

b.)

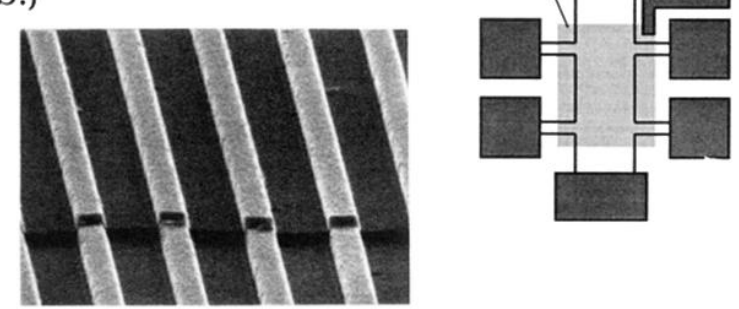

FIG. 1. (a) Sketch of the one-dimensional ferromagnetic Dy grating on top of a $\mathrm{GaAs}-\mathrm{AlGaAs}$ heterojunction. (b) Electron micrograph of the Dy strips evaporated across a mesa edge: $a=1 \mu \mathrm{m}$, height of a Dy strip: $200 \mathrm{~nm}$. (c) Device geometry containing the ferromagnetic grating and an unpatterned reference Hall bar. 